\title{
Conditional Events and Quantum Logic
}

\author{
Philip G. Calabrese \\ Data Synthesis, San Diego, USA \\ Email: pc@datasynthesis.org
}

How to cite this paper: Calabrese, P.G. (2018) Conditional Events and Quantum Logic. Journal of Applied Mathematics and Physics, 6, 1278-1289. https://doi.org/10.4236/jamp.2018.66107

Received: May 2, 2018

Accepted: June 25, 2018

Published: June 28, 2018

Copyright $\odot 2018$ by author and Scientific Research Publishing Inc. This work is licensed under the Creative Commons Attribution International License (CC BY 4.0).

http://creativecommons.org/licenses/by/4.0/

\begin{abstract}
This paper begins with an overview of quantum mechanics, and then recounts a relatively recent algebraic extension of the Boolean algebra of probabilistic events to "conditional events" (order pairs of events). The main point is to show that a so-called "superposition" of two (or more) quantum events (usually with mutually inconsistent initial conditions) can be represented in this algebra of conditional events and assigned a consistent conditional probability. There is no need to imagine that a quantum particle can simultaneously straddle two inconsistent possibilities.
\end{abstract}

\section{Keywords}

Heisenberg Indeterminacy, Superposition of Events, Quantum Entanglement, Hidden Variables, Boolean Algebra, Conditional Logic

\section{Introduction}

Perhaps the first thing to say about quantum mathematics is that it is about variables with a finite number of possible values, not continuous variables. Energy as exhibited by A. Einstein [1] exists and is emitted only in certain fixed quantum amounts or multiples thereof, not in fractional or continuous amounts thereof.

\subsection{Energy Quanta}

For a photon (particle of light), the energy $E$ equals Plank's constant $h$ times the frequency (color) $f$ of the photon. A blue photon with frequency $f_{b}$ has more energy $h f_{b}$ than does a red photon with frequency $f_{r}$ and the positive difference is $\left(f_{b}-f_{r}\right) h$.

Since there must be a smallest energy value $h f_{o}$ for a photon the respective frequencies of the blue and red photons must be multiples of this lowest photon frequency $f_{o}$. 
Apparently, photons carry these different energy values in their axial spin. Although all photons move from place to place in "empty space" at the same speed c, their axial spin frequencies vary by as many as 100 octaves, each octave being twice the frequency (and energy) of the next lower octave. The energy range is proportionately huge. The human eye sees only one octave of color frequencies, but the ear senses lower ones while the whole body feels still lower frequency vibrations.

Although contemporary theory regards photons as having zero mass, they do carry a potential impact as shown by comets; their wispy (gaseous) tails always point away from the sun.

To represent the energy of a spinning or orbiting particle, a vector is needed so as to include both the orientation of the spin (or orbital) axis and also the magnitude of that angular energy of spin (or orbit). And indeed von Neumann and Birkhoff [2] formulated the current standard quantum logic in terms of a vector space-a complete, normed, inner-product space.

\subsection{Heisenberg Indeterminacy}

Another basic principle of quantum mechanics is the Heisenberg Indeterminacy Principal [3]. It arises from the way that present methods of measurements in the quantum world, whether by particle emission or reflection, disturb the quantum object being measured. Therefore, determining the values of certain pairs of variables, like the position and velocity of a particle, requires measurements that typically disturb the object being observed, thereby limiting the precision of the simultaneous measurement of the other variable. A position measurement changes the velocity of the particle; a velocity measurement changes the expected position of the particle.

However, this is a measurement technique ambiguity, purely epistemological. It does not mean that the position and velocity cannot be simultaneously known in principle, just not with the information and measurement methods available. For a particle, various alternate possibilities are mutually exclusive. They are not all "somewhat true" at the same time! Our lack of knowledge about the position and velocity does not imply there is no actual position and velocity. There is no need to imagine some kind of "superposition" of states all of which simultaneously exist with probabilities. But that is what is presently said.

\subsection{Quantum Entanglement}

An important distinction to make in quantum mechanics is between the Heisenberg Indeterminacy Principle and the so-called quantum "entanglement phenomena".

The Double-Slit experiments [4] [5], the Stern-Gerlach spin experiments [6] and John Bell's Theorem [7] as illuminated by N. D. Mermin [8] provide crucial insights into the nature of the mysterious entanglement phenomena of quantum mechanics. In one interpretation, trajectories and spin states of quantum par- 
ticles are influenced by certain energy waves discovered by Schrödinger [9] but later conceptually combined with the associated particles and called "wavicles" and more recently "strings". These later conceptions were designed to instantaneously bridge great distances without influences being conveyed by faster-than-light wave intermediation. Rather than imagining "a faster-than-light, (pre-mass) energy wave reaction to a particle's motion", contemporary theory imagines "a faster-than-light collapse of a space-extended particle-wave from all directions to the location of a measurement". Some kind of instantaneous influence across space is impossible to avoid.

Quantum ambiguities due to Heisenberg's Indeterminacy Principal added to these mysterious measurement correlations of entanglement [10]-[15] and resulted in quantum mechanical "physical states" becoming quantum probability distributions. Physical predictions morphed into probability statements about energy distributions. And that is pretty much where things now stand.

\subsection{Particle Properties and Local Reality}

But does not a particle have an actual position and velocity? According to the de Broglie-Bohm interpretation [12], [15] of Schrödinger's quantum waves, particles have local properties of spin, and the waves merely carry influences at speeds faster than light.

The interference patterns, incrementally assembled by individual particle trajectories through the 2-slit apparatus suggested that somehow each particle was going through both slits! But experiments later confirmed (See [4], pp. 1-7) that each particle indeed went through one but not both slits. Somehow those particles accumulated an interference pattern rather than an independent scatter-shot distribution centered behind each slit.

Combined with Heisenberg Indeterminacy, the ambiguities of entanglement posed a difficult modeling problem. But von J. Neumann knew the experiments so well that he and G. Birkhoff were able to do it with what is now called Hilbert space-a complete, normed, inner product space.

The indeterminacy ambiguity complicated by the entanglement phenomena led to the notion that quantum particles routinely somehow simultaneously occupy two or more mutually inconsistent states. This hypothetical physical state is called a "superposition", a combination of alternate but inconsistent states or trajectories. This prompted that famous question by Einstein et al. [16] about whether a certain cat was dead or alive, or both dead and alive.

\subsection{Conditioning in Logic versus Probability}

The mathematical modeling problem posed by these physical and measurement phenomena was exacerbated by the lack, both in logic and probability theory, of an adequate algebraic conditioning relation. B. Russell's [17] "material implication" over-simplified implication by assuming that a conditional statement that is not false must be true. Although that is adequate for doing 2-valued logic, 
probability theory clearly cannot count as true those instances for which the premise (condition) of a statement is false.

A collection of alternate physical conditions each potentially leading to other specific states has no way of being algebraically expressed in standard Boolean logic nor as an event in standard probability theory. For instance, if $A, B, C$ and $D$ are uncertain probabilistic events, the construction " $(A$ if $B)$ or $(C$ if $D)$ " is neither a Boolean object nor a probabilistic event, but it certainly makes sense as an English statement.

In probability theory, the conditional probability $P(A \mid B)$, of an event $A$ given that an event $B$ also occurs, is defined to be $P(A$ and $B) / P(B)$, and it measures the probability of $A$ in the context of the occurrence of $B$. But the conditional event " $A$ if $B$ " is not an event in standard probability theory, and neither are combinations of such conditionals unless the conditions are equivalent. Therefore both standard Boolean logic and the standard algebra of probabilistic events are missing a 4th operation-conditioning.

Although constructions such as " $A$ if $B)$ or ( $C$ if not $B$ )" have been assigned probabilities in quantum logic, and elsewhere, the underlying algebraic structure has heretofore been incapable of supporting the calculation. So the idea of a "superposition" of mutually inconsistent events was invented as though a particle could somehow simultaneously be in some ambiguous "combination state" of two or more incompatible events, as though the hypothetical cat could be somewhat dead and somewhat alive.

Such mental acrobatics are no longer necessary. The de Broglie-Bohm interpretation of quantum mechanics allows particles to have definite local properties at the cost of including faster-than-light "space force interference reactions" to particle motions in space.

The main purpose of this paper is to show that the so-called superposition of two or more alternative initial conditions (states) can be represented in the author's relatively new algebraic system of "conditional events" [18] and that such constructions can be assigned a consistent probability.

Conditional events are fractions (order pairs) of probabilistic events or Boolean propositions that can consistently carry conditional probabilities. They can also be combined by "or", "and", "not" and "conditioning" like two numerical fractions can be added, multiplied, negated and divided one by the other to get an equivalent fraction. And this extension of Boolean algebra to non-Boolean "conditional events" can represent disjunctions (or) of alternative particle trajectories and assign them consistent probabilities. Thus as far as superposition's are concerned, there is no need to imagine anything more than alternative conditions, only one of which need apply, that is, be factually existent.

\section{The Algebra of Conditional Events (Boolean Fractions)}

Both probability and logic are founded on Boolean algebra: Probabilistic events $A, B, C, \cdots$ form a Boolean algebra just like logical propositions $p, q, r, \cdots$ form a Boolean algebra. Operations of "or", "and" and "not" $(\vee, \wedge$, and ') in logic 
correspond to "union", "intersection" and "complement" $(\cup, \cap$, , ) in probability.

\subsection{A Framework for Logic and Probability}

A model of a given logic is a realization of the axioms of the logic in which each proposition expressible in the logic is either true or false. For instance, a dog is a model (example) of a four-legged animal. A proposition "able to climb trees" may be true in one model and false in another just as a probabilistic event may occur in one instance and not occur in another. However, by the definition of a logical model [19] or probabilistic instance, every proposition is either true in the model or it is false in that model. A probabilistic event $B$ either contains a specific instance (occurs), or it doesn't occur.

For example, the rules of a game of chance, such as coin flipping, define the axioms of a Boolean logic. "If heads you win; if tails you lose." The individual models of this logic are each single coin flip producing "heads", "tails" or "the coin lands on its edge". Note that in each model (flip) the proposition "heads comes up" is either true or it is false.

Or consider the face turned up after the roll of a 6-sided die with numbered faces $\{1,2,3,4,5,6\}$. There are 64 events (or propositions) corresponding to each of the subsets of these 6 numbers. The event (or proposition) that "an odd numbered face comes up" is the subset $\{1,3,5\}$. There are just 6 models or instances, namely the 6 possible faces that can turn up.

This model framework allows the probability of an arbitrary proposition such as "odd face turns up" to be defined as the probability of the set of models in which that proposition is true, namely the probability of the subset $\{1,3,5\}$.

The collection of all subsets of some fixed set forms a Boolean algebra, and these subsets and earlier examples can be assigned probabilities according to the familiar Kolmogorov [20] axioms of a probability measure.

\subsection{Conditional Events and Conditional Propositions}

Analogous to how integer fractions are constructed, ordered pairs of events and associated propositions can be defined.

Let $\mathscr{B}$ be a Boolean algebra of propositions or events, and let $(\mathscr{B} \mid \mathcal{B})$ denote the set of ordered pairs, $\{(a \mid b): a, b$ in $B\}$, called the set of conditionals, "a given $b$ " of $B$. The proposition or event " $b$ " is called the condition or given event, and the proposition or event " $a$ " is called the consequent or conclusion, the proposition " $a$ " in the context of $b$.

\subsubsection{Equivalent Conditionals}

Two conditional statements $(a \mid b)$ and $(c \mid d)$ are defined to be equivalent $(=)$ provided:

1) Their conditions, $b$ and $d$, are equivalent propositions or events; and

2) Their conclusions, $a$ and $c$, are equivalent when their common condition is true. 
In symbols,

$$
(a \mid b)=(c \mid d) \text { if and only if } b=d \text { and } a b=c d
$$

where juxtaposition, " $a b$ ", denotes " $a \wedge b$ ", that is, " $a$ and $b$ ".

In other words, two conditionals are equivalent when they have equivalent premises and their conclusions are equivalent assuming that common premise. This equivalence relation on conditionals implies that for all propositions $a$ and b,

$$
(a \mid b)=(a b \mid b)
$$

and also that for all $a \in \mathscr{B},(1 \mid 0)=(a \mid 0)=(0 \mid 0)$.

$(1 \mid 0)$ is the "inapplicable" or "undefined" conditional and is denoted $U$. The Boolean 1 and 0 propositions are represented by (1|1) and (0|1) respectively.

\subsubsection{Truth-Values of Conditionals}

Thus, in any instance a conditional $(a \mid b)$ can have any one of 3 truth-values:

$(a \mid b)$ is true if $a$ is true and $b$ is true;

$(a \mid b)$ is false if $a$ is false and $b$ is true;

$(a \mid b)$ is inapplicable if $b$ is false.

Thus, $(a \mid b)$ is true on $a \wedge b$, false on $a^{\prime} \wedge b$, and inapplicable on $b^{\prime}$. Note that $(a \mid b)$ has the truth-value of " $a$ " when condition $b$ is true, but is inapplicable when $b$ is false.

We frequently encounter such inapplicable conditional statements or questions in everyday life. Consider a questionnaire that asks, "If you served in the military then were you in the navy?" or "If you own a pet then do you feed table scraps?" These questions cannot be accurately answered "yes" or "no" if their premise (condition) is false any more than an animal lover can answer "yes" or "no" to the question, "Have you stopped beating your dog?" In such a case the answer must be "inapplicable".

\subsection{Operations on Conditionals}

Each of the three operations defined below agrees with the corresponding Boolean operation when applied to conditionals with equivalent conditions. Therefore they extend the Boolean operations.

\subsubsection{Relative Negation (Not)}

The relative negation of "a given $b$ " is the "negation of $a$, given $b$ ". That is,

$$
(a \mid b)^{\prime}=\left(a^{\prime} \mid b\right)
$$

and the latter has probability $1-P(a \mid b)$.

\subsubsection{Disjunction (or)}

The disjunction, " $(a$ if $b$ ) or ( $c$ if $d)$ " of two conditionals means "if either conditional is applicable, i.e. if $(b \vee d)$ is true, then at least one of the two conditionals is true, i.e. ( $a b \vee c d)$ is true", thus, 


$$
(a \mid b) \vee(c \mid d)=((a b \vee c d) \mid(b \vee d))=(a b \vee c d \mid b \vee d)
$$

\subsubsection{Conjunction (and)}

The conjunction, " $a$ if $b$ and $c$ if $d$ " means "if either conditional is applicable then one is true while the other is not false". That is,

$$
\begin{gathered}
(a \mid b) \wedge(c \mid d)=\left[a b\left(c \vee d^{\prime}\right) \vee\left(a \vee b^{\prime}\right) c d\right] \mid(b \vee d) \\
=\left(a b d^{\prime} \vee a b c d \vee b^{\prime} c d \mid b \vee d\right)
\end{gathered}
$$

which also means "if either conditional is applicable then either they are both true or else one is true while the other is inapplicable."

\subsubsection{Conditional Conditionals}

In practice, adding a condition " $c$ " to a conditional $(a \mid b)$ merely means $(a \mid b c)$. That is,

$$
((a \mid b) \mid c)=(a \mid b c)
$$

Therefore the iterated conditional

$$
((a \mid b) \mid(c \mid d))=(a \mid b \wedge(c \mid d))=\left(a \mid b\left(c \vee d^{\prime}\right)\right)
$$

because by the operations $b \wedge(c \mid d)=(b \mid 1) \wedge(c \mid d)=\left(b\left(c \vee d^{\prime}\right) \mid 1\right)=b\left(c \vee d^{\prime}\right)$.

That is, " $(a \mid b)$ if $(c \mid d)$ " means " $a$ if [ $b$ is true and $(c \mid d)$ is not false]".

The order of preference of the operations ${ }^{1}$ is negation (') before conjunction $(\wedge)$, before disjunction $(\vee)$, before conditioning $(\mid)$.

The algebra $(\mathscr{B} \mid \mathcal{B})$ of conditionals includes the original Boolean algebra $\mathscr{B}$ as those conditionals ( $a \mid \Omega$ ), where $\Omega$ is the universal event, and " $a$ " is any member of $\mathcal{B}$. In logical notation these are the conditionals $(a \mid 1)$ whose condition is certain.

\subsection{Indicator Function Representation of Events}

It is helpful to visualize events and conditional events as indicator functions; see Figure 1. Suppose $\mathbb{P}=(\Omega, \mathscr{B}, P)$ is a probability space of individual instances $\Omega$, events $\mathcal{B}$ (an algebra of subsets of instances), and probability measure $P$. Then the characteristic function of each measurable subset $B, B \in \mathscr{B}$ is a unique measurable indicator function $q_{B}: \Omega \rightarrow\{0,1\}$ from $\Omega$ into the 2-element Boolean algebra $\{0,1\}$ defined as follows:

$$
\left(q_{B}\right)(\omega)= \begin{cases}1, & \text { if } \omega \in B \\ 0, & \text { if } \omega \in B^{\prime}\end{cases}
$$

The function $q$ (dropping the subscript) is a "proposition" in the sense that for each $\omega \in \Omega$, either $q$ is true for $\omega$, meaning $q(\omega)=1$, or else $q$ is false for $\omega$, meaning $q(\omega)=0$. $L$ will denote the set of all propositions of $\mathscr{P}$. Conversely, each measurable indicator function $q$ defines a unique measurable subset $B, B \in \mathcal{B}$ by

${ }^{1}$ These operations and the resulting not-altogether-Boolean algebra of conditional events are completely characterized in [18], p. 56. 


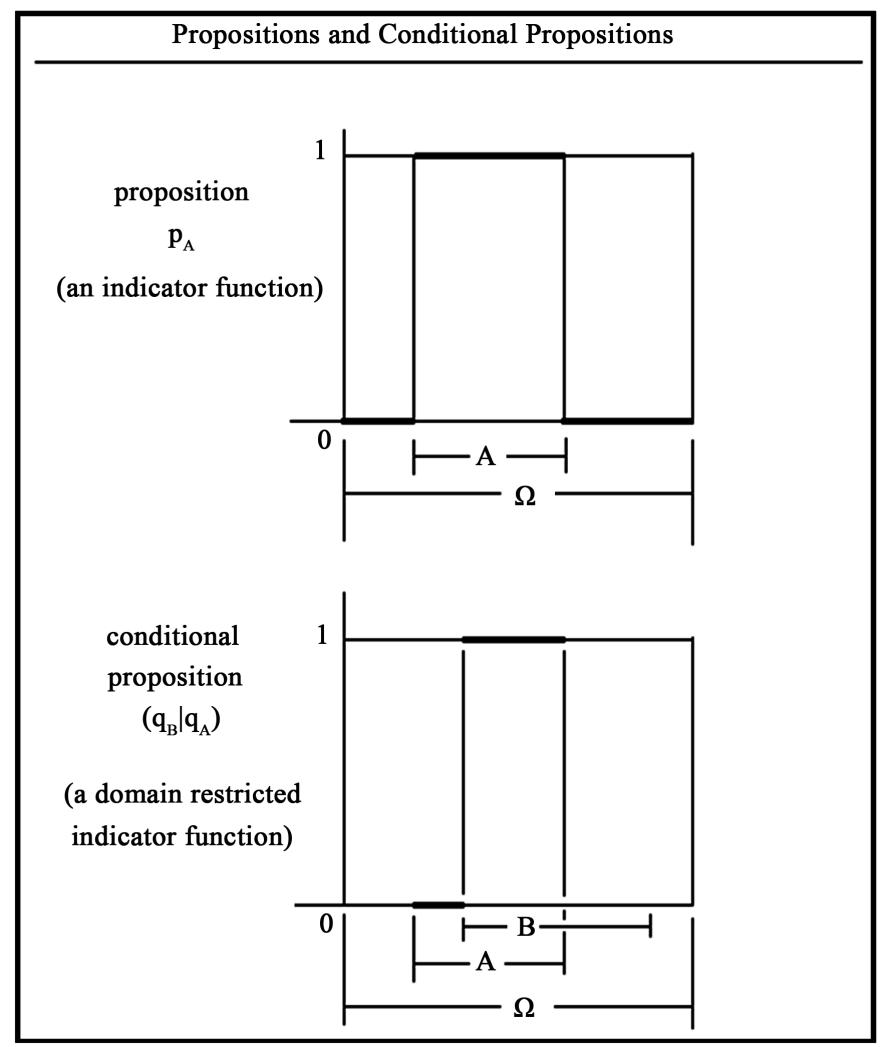

Figure 1. Propositions and conditional propositions.

$$
B=q^{-1}(1)=\{\omega \in \Omega: q(\omega)=1\}
$$

$B$ is the measurable subset of cases (instances) for which $q$ is true, and $P(B)$ is the probability measure of the partial truth of $q$.

\subsection{Indicator Function Representation of Events}

Each ordered pair, $(B \mid A)$ of measurable subsets $B, A$ in $B$ with corresponding indicator functions $q$, $p$, defines a unique domain-restricted measurable indicator function ( $q \mid p): A \rightarrow\{0,1\}$, from $A$ into the 2-element Boolean algebra as follows:

$$
(q \mid p)(\omega)= \begin{cases}1, & \text { if } \omega \in B \cap A \\ 0, & \text { if } \omega \in B^{\prime} \cap A \\ U, & \text { if } \omega \in A^{\prime}\end{cases}
$$

where $U$ means "undefined". This can be expressed in terms of the unconditioned propositions $p$ and $q$ by

$$
(q \mid p)(\omega)= \begin{cases}q(\omega) & \text { if } p(\omega)=1 \\ U & \text { if } p(\omega)=0\end{cases}
$$

$(q \mid p)$ is a "conditional proposition" in the sense that if $p$ is true for $\omega$ then either $(q \mid p)$ is true for $\omega$ or $(q \mid p)$ is false for $\omega$. On the other hand, we say that $(q \mid p)$ "does not apply" (i.e., is undefined or inapplicable) for those $\omega$ for which $p$ is false. Thus $(q \mid p)$ has three truth states. $(q \mid p)$ is simply $q$, restricted to $p^{-1}(1)$, the 
subset on which $p$ is true.

\section{Quantum Conditioning}

Besides being able to characterize simultaneously verifiable quantum events and a number of other properties of quantum logic, the conditional event algebra ${ }^{2}$ that I am recommending can disambiguate the notion of a superposition of quantum events.

\subsection{Superpositions Expressed as the Disjunctions of Conditional Events}

Consider a quantum system and associated measurement apparatus that starts out in a condition (state) $d$, and after some one of a number of mutually exclusive and exhaustive events $\left\{b_{i}\right\}$ occur and we observe the value of some proposition $c$. In the standard Hilbert linear space calculation, the "amplitude" or logical representative of observing $c$ via the $b_{i}$ starting with condition $d$ is

$$
\langle c \mid d\rangle=\sum_{i}\left\langle c \mid b_{i}\right\rangle\left\langle b_{i} \mid d\right\rangle
$$

and the probability of this observation is the square of the norm of the vector $\langle c \mid d\rangle$

$$
P\langle c \mid d\rangle=\left|\sum_{i}\left\langle c \mid b_{i}\right\rangle\left\langle b_{i} \mid d\right\rangle\right|^{2}
$$

In the realm of conditionals this formula is first expressed as

$$
(c \mid d)=\vee_{i}\left(c \mid b_{i} d\right)\left(b_{i} d \mid d\right)
$$

with probability

$$
P(c \mid d)=\sum_{i} P\left(c \mid b_{i} d\right) P\left(b_{i} d \mid d\right) .
$$

However, in the quantum physical situation described above, starting from an initial condition $d$, the subsequent occurrence of an intermediate event $b_{i}$ usually makes additional subsequent events such as $c$ independent of $d$, which can be expressed as $P\left(c \mid b_{i} d\right)=P\left(c \mid b_{i}\right)$. So

$$
P(c \mid d)=\sum_{i} P\left(c \mid b_{i}\right) P\left(b_{i} \mid d\right)
$$

This follows by substitution and since $P\left(b_{i} d \mid d\right)=P\left(b_{i} \mid d\right)$.

In the vernacular of quantum mechanics, $\left(c \mid b_{i}\right)$ is the "projection" of $c$ onto the Boolean subspace $\left(\mathscr{B} \mid b_{i}\right)$ and $P\left(b_{i} \mid d\right)$ is the relative probability of the Boolean subspace generated by $b_{i}$ given $d$.

Notice that in $(\mathscr{B} \mid \mathcal{B})$ so-called "superpositions" can be expressed as disjunctions of quantum events that have incompatible conditions. For instance, $(a \mid b d) \vee\left(c \mid b^{\prime} d\right)$ exists and has a consistent probability, namely $P\left(a b d \vee c b^{\prime} d \mid b d \vee b^{\prime} d\right)=P\left(a b \vee c b^{\prime} \mid d\right)$. There is no need to believe that this situation is any more mysterious than two conditional alternatives, only one of which applies.

${ }^{2}$ See Chapters 8 and 9 of [18] for a fuller account of how quantum logic can be expressed in the logic of conditional events. 


\subsection{Quantum Vectors and Linear Operator Language}

The summation $\sum_{i} P\left(c \mid b_{i}\right) P\left(b_{i} \mid d\right)$ can be written as the inner product $(\bullet)$ of the two countable vectors

$$
\left\langle P\left(c \mid b_{1}\right), P\left(c \mid b_{2}\right), \cdots, P\left(c \mid b_{i}\right), \cdots\right\rangle \cdot\left\langle P\left(b_{1} \mid d\right), P\left(b_{2} \mid d\right), \cdots, P\left(b_{i} \mid d\right), \cdots\right\rangle
$$

or two finite vectors in case $\left\{b_{i}\right\}$ is a finite partition of the universe of possibilities.

An inner product of two n-dimensional real vectors can be interpreted as the product of their lengths times the cosine of the angle between them, and the absolute value of this is the length of one vector times the length of the projection of the 2 nd vector onto the first.

The first vector can be considered to be the result of the "projection operator" $\left[P\left(\cdot \mid b_{1}\right), P\left(\cdot \mid b_{2}\right), \cdots, P\left(\cdot \mid b_{i}\right), \cdots\right]$ applied to any proposition $c$. It gives the vector of projection (conditional) probabilities of any proposition $c$ on (given) the basis propositions $\left\{b_{i}: i=1,2, \cdots\right\}$.

The second vector is the vector of relative probabilities of the basis propositions given some initial state (proposition) $d$. The sum over $i$ of the probabilities $P\left(b_{i} \mid d\right)$ is 1 . The probability of $c$ is therefore a linear combination of the individual conditional probabilities of $c$ given $b_{i}$. If nothing is known about the initial state, then $d=1$, and so the second vector becomes the vector $\left\langle P\left(b_{1}\right), P\left(b_{2}\right), \cdots, P\left(b_{i}\right), \cdots\right\rangle$ of relative probabilities of the basis propositions, and the equation reduces to

$$
P(c)=\sum_{i} P\left(c \mid b_{i}\right) P\left(b_{i}\right) .
$$

\subsection{Quantum Measurements Perturbations versus Passive Conditioning}

In the above, $P(d \mid d)$ represents the probability of a quantum state $d$ that without further measurement perturbation will always be found in state $d$, and of course that probability is 1 . But when, for instance, there is an assumed amplitude from a state $d$ to each member of a complete set of orthogonal states $\left\{b_{i}\right\}$ and then another amplitude back to state $d$ in a subsequent perturbation of the system, the probability $P($ not $d \mid d)$ need not be 0 as it must if $P(d \mid d)=1$. In this double transformation case $P\left(d^{\prime} \mid d\right)=\sum_{i} P\left(d^{\prime} \mid b_{i}\right) P\left(b_{i} \mid d\right)$, which need not be zero because the probability distribution (state) has changed. This illustrates why the designers of quantum logic decided to define a "state of the system" as a probability distribution on vectors, vectors built from atoms of complete assignments of values to variables. Every measurement of a variable in general produces a new state (condition) of the probability distribution for the system.

This situation is not yet covered by a theory of conditioning that only accumulates conditions and assumes there is no additional measurement disturbance but merely seeks to answer the probability questions of what else might be true given that the condition is true.

Nevertheless, even though the measurements are perturbation operators on 
the probability distribution being measured, the new post-disturbed probability distributions (quantum states) are still defined in $(\mathscr{B} \mid \mathscr{B})$ as sub-spaces $(\mathscr{B} \mid b)$ generated by alternative possible conditions $b$ given to be true in the new state.

\section{Conclusion}

The so-called "superposition" of a quantum particle in two possibly mutually inconsistent quantum states $B, D$ can be represented in the new extension algebra [18] of conditional events as the event $A$ given condition $B$ or event $C$ given the alternate condition $D$. This non-Boolean object $((A \mid B) \cup(C \mid D))$ is a member of the new algebra, namely $((A \cap B) \cup(C \cap D) \mid(B \cup D))$, and it can be assigned the consistent conditional probability, $P((A \cap B) \cup(C \cap D) \mid(B \cup D))$. The quantum particle can be imagined as being in one or the other of the initial conditions without being in both.

\section{References}

[1] Einstein, A. (1906) Zur Theorie der Lichterzeugung und Lichtabsorption [On the Theory of Light Production and Light Absorption]. Annalen der Physik, 325, 199-206. https://doi.org/10.1002/andp.19063250613

[2] Birkhoff, G. and von Neumann, J. (1936) The Logic of Quantum Mechanics. Annals of Mathematics, 37, 823-834. https://doi.org/10.2307/1968621

[3] Heisenberg, W. (1930) The Physical Principles of the Quantum Theory. Dover Publications, Dover.

[4] Feynman, R.P., Leighton, R.B. and Sands, M. (1965) Lectures on Physics, Vol. III: Quantum Mechanics. Addison-Wesley Publishing Co., Barrington.

[5] Koopman, B.O. (1955) Quantum Theory and the Foundations of Probability. In: MacColl, L.A., Ed., Applied Probability, McGraw-Hill Education, New York, 97-102.

[6] https://en.m.wikipedia.org/wiki/Stern-Gerlach_experiment

[7] Bell, J.S. (1964) On the Einstein-Podolsky-Rosen Paradox. Physical Review Journals, 1, 195-200.

[8] Mermin, N.D. Department of Physics Cornell Arts and Sciences. https://www.google.com/search?q=n.+david+mermin\&ie=utf-8\&oe=utf-8\&client=f irefox-b-1

[9] Schrödinger, E.R. (1926) An Undulatory Theory of the Mechanics of Atoms and Molecules. Physical Review, 28, 1049-1070. https://doi.org/10.1103/PhysRev.28.1049

[10] de Broglie, L.V. (1923) Radiations-Ondes et Quanta/Radiation-Waves and Quanta. Comptes Rendus Mathématique, 177, 507-510.

[11] Bell, J.S. (1966) On the Problem of Hidden Variables in Quantum Mechanics. Reviews of Modern Physics, 38, 447-452. https://doi.org/10.1103/RevModPhys.38.447

[12] Bohm, D.J. (1983) Wholeness and the Implicate Order. Ark Paperbacks, London.

[13] Goldstein, S. (1996) Bohmian Mechanics and the Quantum Revolution. http://arxiv.org/abs/quant-ph/9512027

[14] Goldstein, S. (1996) Quantum Philosophy: The Flight from Reason in Science. In: Gross, P., Levitt, N. and Lewis, M.W., Eds., The Flight from Science and Reason. 
http://arxiv.org/abs/quant-ph//9601007

[15] Goldstein, S. (2002) Bohmian Mechanics. In: Zalta, E.N., Ed., The Stanford Encyclopedia of Philosophy.

http://plato.stanford.edu/archives/win2002/entries/qm-bohm

[16] Einstein, A., Podolsky, B.Y. and Rosen, N. (1935) Can Quantum-Mechanical Description of Physical Reality Be Considered Complete? Physical Review, 47, 777-780. https://doi.org/10.1103/PhysRev.47.777

[17] Russell, B.A.W. and Whitehead, A.N. (1913) Principia Mathematica. Vol. 3, Cambridge University Press, Cambridge.

[18] Calabrese, P.G. (2017) Logic and Conditional Probability-A Synthesis. College Publications.

[19] Chang, C.C. and Keisler, H.J. (1973) Model Theory. 2nd Edition, Amsterdam.

[20] Kolmogorov, A.N. (1933) Foundations of the Theory of Probability. 2nd Edition, Chelsea Publishing Company, New York. 